This is the post peer-review accepted manuscript of:

M. Rusci, L. Cavigelli and L. Benini, "Design Automation for Binarized Neural Networks: A Quantum Leap Opportunity?" 2018 IEEE International Symposium on Circuits and Systems (ISCAS), Florence, Italy, 2018, pp. 1-5. doi: 10.1109/ISCAS.2018.8351807

The published version is available online at: https://doi.org/10.1109/ISCAS.2018.8351807

(C) 2018 IEEE. Personal use of this material is permitted. Permission from IEEE must be obtained for all other uses, in any current or future media, including reprinting/republishing this material for advertising or promotional purposes, creating new collective works, for resale or redistribution to servers or lists, or reuse of any copyrighted component of this work in other works 


\title{
Design Automation for Binarized Neural Networks: A Quantum Leap Opportunity?
}

\author{
Manuele Rusci*, Lukas Cavigelli ${ }^{\dagger}$, Luca Benini ${ }^{\dagger *}$ \\ * Energy-Efficient Embedded Systems Laboratory, University of Bologna, Italy - manuele.rusci@unibo.it \\ ${ }^{\dagger}$ Integrated Systems Laboratory, ETH Zurich, Switzerland - \{cavigelli, benini\}@iis.ee.ethz.ch
}

\begin{abstract}
Design automation in general, and in particular logic synthesis, can play a key role in enabling the design of application-specific Binarized Neural Networks (BNN). This paper presents the hardware design and synthesis of a purely combinational BNN for ultra-low power near-sensor processing. We leverage the major opportunities raised by BNN models, which consist mostly of logical bit-wise operations and integer counting and comparisons, for pushing ultra-low power deep learning circuits close to the sensor and coupling them with binarized mixed-signal image sensor data. We analyze area, power and energy metrics of BNNs synthesized as combinational networks. Our synthesis results in GlobalFoundries $22 \mathrm{~nm}$ SOI technology shows a silicon area of $2.61 \mathrm{~mm}^{2}$ for implementing a combinational $\mathrm{BNN}$ with $32 \times 32$ binary input sensor receptive field and weight parameters fixed at design time. This is $2.2 \times$ smaller than a synthesized network with re-configurable parameters. With respect to other comparable techniques for deep learning near-sensor processing, our approach features a $10 \times$ higher energy efficiency.
\end{abstract}

\section{INTRODUCTION}

Bringing intelligence close to the sensors is an effective strategy to meet the energy requirements of battery-powered devices for always-ON applications [1]. Power-optimized architectures for near-sensor processing target the reduction of the amount of data to be dispatched out from the sensors, along with significant energy savings, by applying local data processing over raw sensed data [2]. To this aim, in the context of visual sensing, novel computer vision chips include insensor computational modules for the early extraction of midand low- level visual features, which are transferred to a digital processing unit for further computation or used to feed a first stage classifier [3]. Thanks to the integration of analog processing circuits on the focal-plane, the amount of data crossing the costly analog-to-digital border is reduced [4] and, if compared with a camera-based system featuring a traditional imaging technology, the system energy consumption is lower because of (a) a reduced sensor-to-processor bandwidth and (b) a lower demand for digital computation [5]. Relevant examples of mixed-signal computer vision capabilities include the extraction of spatial and temporal features, such as edges or frame-difference maps, or a combination of them [6]. Because of the employed highly optimized architectures, the power consumption of these smart visual chips results to be more than one order of magnitude lower than off-the-shelf traditional image sensors [7].

However, to favor the meeting between smart ultra-low power sensing and deep learning, which is nowadays the leading technique for data analytics, a further step is required. At present, the high computational and memory requirement of deep learning inference models have prevented a full

This project was supported in part by the EU's H2020 programme under grant no. 732631 (OPRECOMP) and by the Swiss National Science Foundation under grant 162524 (MicroLearn). integration of these approaches close to the sensor at an ultra low power cost [4], [8]. A big opportunity for pushing deep learning into low-power sensing come from recently proposed Binarized Neural Networks (BNNs) [9], [10]. When looking at the inference task, a BNN consists of logical XNOR operations, binary popcounts and integer thresholding. Therefore, major opportunities arise for hardware implementation of these models as part of the smart sensing pipeline [11].

In this paper, we explore the feasibility of deploying BNNs as a front-end for an ultra-low power smart vision chip. The combination of mixed-signal processing and hardware BNN implementation represents an extremely energy-efficient and powerful solution for always-ON sensing, serving as an early detector of interesting events. Therefore, we design and synthesize a purely combinational hard-wired BNN, which is fed with the binary data produced by a mixed-signal ultra-low power imager [7]. The main contributions of this paper are:

- The hardware design and logic synthesis of a combinational $\mathrm{BNN}$ architecture for always-ON near-sensor processing.

- The area and energy evaluation of the proposed approach, for varying network models and configurations.

We evaluate two BNN models with $16 \times 16$ and $32 \times 32$ binary input size, either with fixed or variable parameters. In case of a combinational $\mathrm{BNN}$ with $32 \times 32$ input data and hardwired parameters, our synthesis results in GlobalFoundries $22 \mathrm{~nm}$ SOI technology shows an area occupancy of $2.61 \mathrm{~mm}^{2}$, which is $2.2 \times$ smaller than the model with variable parameters, and features a $10 \times$ higher energy efficiency with respect to comparable techniques for deep learning-based near-sensor processing. Moreover, our study paves the way for exploring a new generation of logic synthesis tools-aimed at aggressively optimizing deep binarized networks and enabling focal-plane processing of images with higher resolution.

\section{RELATED WORK}

Besides the mixed-signal processing circuits for extracting basic spatial and temporal visual features in-the-sensor [6], [12], [13], recent approaches tried to push deep learning circuits into the analog side to exploit the energy benefits of focal-plane processing [3]. The work presented in [14] makes use of angle-sensitive pixels, integrating diffraction gratings on the focal plane. Based on the different orientations of the pixellevel filters, multiple feature maps are locally computed as the first layer of a convolutional network. RedEye [4] embeds column-wise processing pipelines in the analog domain to perform 3D convolutions before of the digital conversion. The chip is implemented in $0.18 \mu \mathrm{m}$ technology and needs $1.4 \mathrm{~mJ}$ to process the initial 5 layers of GoogLeNet, leading to an energy efficiency of about $2 \mathrm{TOp} / \mathrm{s} / \mathrm{W}$. A sensing front-end supporting analog multiplication is proposed in [15] to operate 
on analog sensed data. The authors introduce a MAC unit composed of only passive switches and capacitors to realize a switched-capacitor matrix multiplier, which achieves an energy efficiency of $8.7 \mathrm{TOp} / \mathrm{s} / \mathrm{W}$ when running convolution operations. With respect to these analog approaches, we leverage the potentiality of BNNs to deploy a digital and optimized purely combinational network to notably increase the energy efficiency of near-sensor processing circuits.

On the near-sensor digital side, many neural network accelerators have been reported in the literature, most of them with an energy efficiency in the range of few TOp/s/W [16][18]. Several recent approaches have focused on quantizing the weights down to binarization in order to gain a significant advantage in memory usage and energy efficiency [8], [18], pushing it up to around $60 \mathrm{TOp} / \mathrm{s} / \mathrm{W}$ while advances in training methods have achieved accuracy losses of less than $1 \%$ for this setup. A new approach has been to quantize also the activations down to binary with initial accuracy losses of up to $30 \%$ on the ILSVRC dataset, these have improved to around $11 \%$ over the last two years and even less for smaller networks on datasets such as CIFAR-10 and SVHN [9], [10], [18]. During this time, some VLSI implementations have been published, most of them targeting FPGAs such as the FINN framework [11], [19]. Only few ASIC implementations exist [19]-[21], of which XNOR-POP uses in-memory processing and reports the highest energy efficiency of $22.2 \mathrm{TOp} / \mathrm{s} / \mathrm{W}$ and thus less than the best binary-weight-only implementation.

\section{Combinational Hardware BNN DESIGN}

A BNN design represents both the networks weights and the activation layers with single-bit precision, leading to an intrinsic $32 \times$ memory footprint reduction with respect to a baseline full-precision model. When applying the binarization scheme to a Convolutional Neural Network (CNN), the resulting BNN features a stacked architecture of binary convolutional layers, where every layer transforms $I F$ binary input feature maps into $O F$ binary output feature maps through the well-known convolution operation. Because of the binary domain, denoted as $\{0,1\}$, of both the input data and the weight filters, the convolution kernel can be rewritten as

$\varphi(m, x, y)=\operatorname{popcount}($ weights $(m)$ xnor recField $(x, y)),(1)$ where $\varphi(m, x, y)$ is the result of the convolution, weights $(m)$ is the array of binary filter weights and recField $(x, y)$ is the receptive field of the output neuron located at position $(x, y)$ of the $m$-th output feature map. The popcount $(\cdot)$ function returns the numbers of asserted bits of the argument. Note that the convolution output $\varphi(m, x, y)$ is an integer value. As presented by [9], the popcount result is binarized after a batch normalization layer. However, the normalization operation can be reduced to a comparison with an integer threshold,

$$
\operatorname{outMap}(m, x, y)=\left\{\begin{array}{ll}
\varphi(m, x, y) \geq \operatorname{thresh}(m) & \text { if } \gamma>0 \\
\varphi(m, x, y) \leq \operatorname{thresh}(m) & \text { if } \gamma<0 \\
1 & \text { if } \gamma=0 \text { and } \beta \geq 0 \\
0 & \text { if } \gamma=0 \text { and } \beta<0
\end{array}\right. \text {, }
$$

where thresh $(m)$ is the integer threshold that depends on the convolution bias $b$ and on the parameters learned by the batch normalization layer $\mu, \sigma, \gamma$ and $\beta$. After training the network, the $\operatorname{thresh}(m)$ parameters are computed offline as $\lfloor\mu-b-$ $\beta \cdot \sigma / \gamma\rfloor$ if $\gamma>0$ or $[\mu-b-\beta \cdot \sigma / \gamma]$ if $\gamma<0$.

Fig. 1 graphically schematizes the binary convolution operation. The BinConv module applies (1) and (2) over the

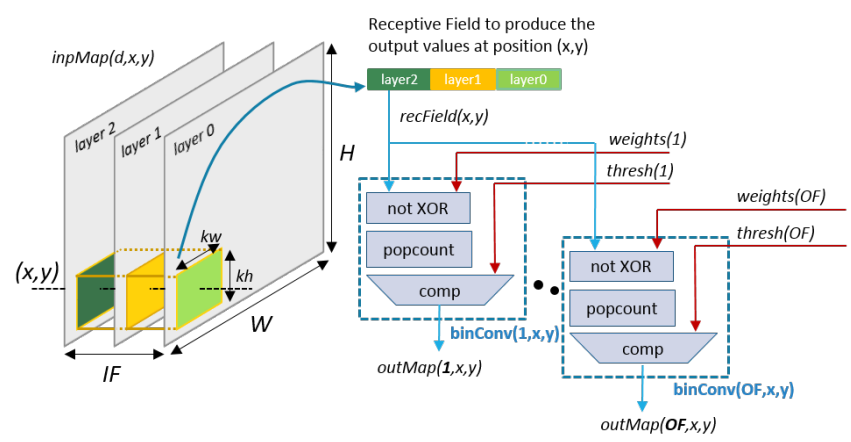

Fig. 1. Computational flow of a binary convolutional layer. For each of the OF output feature maps, the binary value at position $(x, y)$ is produced by overlapping the $m$-th weight filter to the array of the receptive field of the input feature map centered at the spatial position $(x, y)$.

receptive field values associated to any of the output neurons outMap $(m, x, y)$. Hence, to build a convolutional layer, the BinConv is replicated for every output neuron. The hardware architecture of a BinConv element is shown in Fig. 2. In the figure, the input signals recField $(x, y)$, weights $(m)$ and $\operatorname{thresh}(m)$ and the output signal outMap $(m, x, y)$ of the block refer to (1) and (2). Additionally, the $\operatorname{sign}(m)$ signal drives the selection of the correct output neuron's value depending on the batch normalization parameters (eq. (2)). The network parameters, weights, thresh and sign, highlighted in red, can be stored in a memory block, to allow online reconfiguration, or can be fixed at design time. In the first case, the memory footprint required to store the parameters of a convolutional layer is $O F \cdot\left(I F \cdot k w \cdot k h+\left\lfloor\log _{2}(I F \cdot k w \cdot k h)\right\rfloor+3\right)$ bits. On the contrary, if hard-wiring the binary weights, the circuit implementation lacks flexibility but benefits in terms of silicon occupation.

To explore the feasibility of deep combinational BNNs, we focus on VGG-like network topologies as in [9]. These networks include convolutional layers with a small filter size (typically $k w=k h=3$ ) and an increasing feature dimension going deeper into the network. The spatial dimension tends to decrease by means of strided pooling operations placed after the binary convolution of (1). Following the intuition of [11], a MaxPooling layer can be moved behind the binarization by replacing the MAX with an OR operation among the binary values of the activations. The network frontend is composed by multiple fully-connected layers. Their hardware implementation is similar to the binConv module of Fig. 2, where the convolutional receptive field contains all the input neurons of the layer. The last fully-connected layer generates a confidence score for every class. Differently from the original BNN scheme, our network architecture is fed with a binary

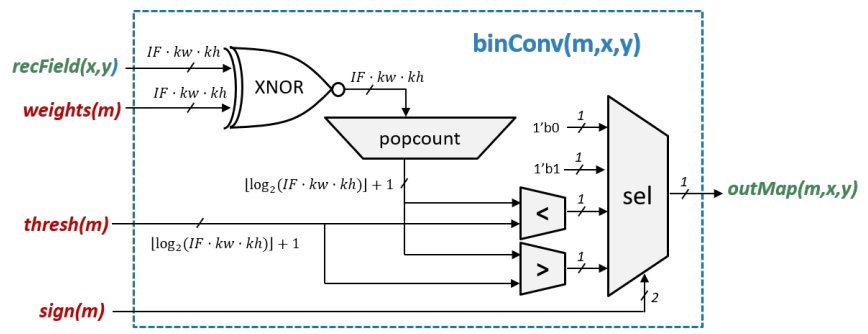

Fig. 2. Hardware architecture of the combinational building block for computing binary convolutions. Every $\operatorname{bin} \operatorname{Conv}(m, x, y)$ module instantiated within a convolutional layer produces the binary value of the output neuron at location $(x, y)$ of the $m$-th output feature map. 
TABLE I

VGG-LIKE BNN MODELS

\begin{tabular}{|c|c|c|}
\hline layer & Model with a $16 \times 16$ input map & Model with a $32 \times 32$ input map \\
\hline 1 & bConvLyr3x3( 1,16)+MaxP2x2 & bConvLyr3x3( 1,16$)+\mathrm{MaxP} 2 \times 2$ \\
\hline 2 & bConvLyr $3 \times 3(16,32)+$ MaxP2 22 & bConvLyr $3 \times 3(16,32)+\operatorname{MaxP} 2 \times 2$ \\
\hline 3 & bConvLyr3x3(32,48)+MaxP2x2 & bConvLyr3x3(32,48)+MaxP2x2 \\
\hline 4 & bFcLyr $(192,64)$ & bConvLyr $3 \times 3(48,64)+\operatorname{MaxP} 2 \times 2$ \\
\hline 5 & bFcLyr $(64,4)$ & bFcLyr $(256,64)$ \\
\hline 6 & & bFcLyr $(64,4)$ \\
\hline
\end{tabular}

single-layer signal coming from a mixed-signal imager [7]. However, the presented approach also holds for multi-channel imagers.

\section{A. Estimating Area}

Before looking at synthesis results, we estimate the area of a binary convolutional layer. For each output value (output pixel and feature map, $\left.N_{\text {out }}=H \cdot W \cdot O F\right)$, we have a receptive field of size $N_{\mathrm{RF}}=I F \cdot k w \cdot k h$ and thus need a total of $N_{\text {out }} N_{\mathrm{RF}}$ XNOR gates. These are followed by popcount units-adder trees summing over all $N_{\mathrm{RF}}$ values in the receptive field. The resulting full-precision adder trees require $\sum_{i=1}^{\log _{2}\left(N_{\mathrm{RF}}\right)} N_{\mathrm{RF}} 2^{-i}=N_{\mathrm{RF}}-1$ half-adders and $\sum_{i=1}^{\log _{2}\left(N_{\mathrm{RF}}\right)}(i-1) N_{\mathrm{RF}} 2^{-i}=N_{\mathrm{RF}}-\log _{2}\left(N_{\mathrm{RF}}\right)-1$ fulladders (FAs) each, and are replicated for every output value. The subsequent threshold/compare unit is insignificant for the total area.

To provide an example, we look at the first layer of the network for $16 \times 16$ pixel images with 1 input and 16 output feature maps and a $3 \times 3$ filter $\left(N_{\mathrm{RF}}=9, N_{\text {out }}=4096\right)$. Evaluating this for the GF22 technology with $A_{\mathrm{XNOR}}=0.73 \mu \mathrm{m}^{2}$, $A_{\mathrm{HA}}=1.06 \mu \mathrm{m}^{2}$ and $A_{\mathrm{FA}}=1.60 \mu \mathrm{m}^{2}$, we obtain an area of $A_{\mathrm{XNOR}, \text { tot }}=0.027 \mathrm{~mm}^{2}, A_{\mathrm{HA}, \text { tot }}=0.033 \mathrm{~mm}^{2}$ and $A_{\mathrm{FA}, \text { tot }}=0.029 \mathrm{~mm}^{2}$-a total of $0.089 \mathrm{~mm}^{2}$. Note that this implies that the area scales faster than linearly with respect to the size of the receptive field $N_{\mathrm{RF}}$ since the word width in the adder tree increases rapidly. This is not accounted for in the widely used GOp/img complexity measure for NNs, as it is becoming only an issue in this very low word-width regime.

\section{EXPERIMENTAL RESULTS}

\section{A. BNN Training}

The experimental analysis focuses on two VGG-like network topologies described in Tbl. I to investigate the impact of different input and network size. As a case-study, we trained the networks with labelled patches from the MIOTCD dataset [22] belonging to one of the following classes: car, pedestrian, cyclist and background. The images from the dataset are resized to fit the input dimension before applying a non-linear binarization, which simulates the mixed-signal preprocessing of the sensor [7]. By training the BNNs with ADAM over a training set of about 10ksamples/class (original images are augmented by random rotation), the classification accuracy against the test-set achieves $64.7 \%$ in case of the model with $32 \times 32$ input data, while a $50 \%$ is measured for the $16 \times 16$ model because of the smaller input size and network. Since this work focuses on hardware synthesis issues of BNN inference engines, we do not explore advanced training

\footnotetext{
${ }^{1}$ bConvLyr $3 \times 3(x, y)$ indicates a binary convolutional layer with a $3 \times 3$ filter $x$ input and $y$ output feature maps, MaxP2x2 is a max pooling layer of size $2 \times 2, \operatorname{bFcLyr}(x, y)$ is a binary fully connected layer with $x$ binary input $y$ binary output binary neurons.
}

TABLE II

SYNTHESIS AND POWER RESULTS FOR DIFFERENT CONFIGURATIONS

\begin{tabular}{|c|c|c|c|c|c|c|c|c|}
\hline \multirow[b]{2}{*}{ netw. } & \multirow[b]{2}{*}{ type } & \multicolumn{2}{|c|}{- area -} & \multicolumn{2}{|c|}{ — time/img - } & \multirow{2}{*}{$\begin{array}{r}\text { E/img } \\
{[\mathrm{nJ}]}\end{array}$} & \multirow{2}{*}{$\begin{array}{l}\text { leak. } \\
{[\mu \mathrm{W}]}\end{array}$} & \multirow{2}{*}{$\begin{array}{r}\text { E-eff. } \\
{[\mathrm{TOp} / \mathrm{J}]}\end{array}$} \\
\hline & & {$\left[\mathrm{mm}^{2}\right]$} & {$[\mathrm{MGE}]^{\dagger}$} & [ns] & {$[\mathrm{FO} 4]^{\ddagger}$} & & & \\
\hline $16 \times 16$ & var. & 1.17 & 5.87 & 12.82 & 560 & 2.40 & 945 & 470.8 \\
\hline $16 \times 16$ & fixed & 0.46 & 2.32 & 12.40 & 541 & 1.68 & 331 & 672.6 \\
\hline $32 \times 32$ & var. & 5.80 & 29.14 & 17.27 & 754 & 11.14 & 4810 & 479.4 \\
\hline $32 \times 32$ & fixed & 2.61 & 13.13 & 21.02 & 918 & 11.67 & 1830 & 457.6 \\
\hline
\end{tabular}

TABLE III

AREA BREAKDOWN FOR THE $16 \times 16$ NETWORK

\begin{tabular}{cr|rrr}
\hline layer & $\begin{array}{r}\text { compute } \\
{[\mathrm{kOp} / \mathrm{img}]}\end{array}$ & $\begin{array}{r}\text { area estim. } \\
{\left[\mathrm{mm}^{2}\right]}\end{array}$ & $\begin{array}{r}\text { var. weights } \\
\text { area }\left[\mathrm{mm}^{2}\right]\end{array}$ & $\begin{array}{r}\text { fixed weights } \\
\text { area }\left[\mathrm{mm}^{2}\right]\end{array}$ \\
\hline 1 & $74(6.5 \%)$ & 0.093 & $0.077(6.6 \%)$ & $0.008(1.7 \%)$ \\
2 & $590(52.2 \%)$ & 0.971 & $0.647(55.4 \%)$ & $0.204(44.3 \%)$ \\
3 & $442(39.1 \%)$ & 0.738 & $0.417(35.8 \%)$ & $0.241(52.3 \%)$ \\
4 & $25(2.2 \%)$ & 0.041 & $0.026(2.2 \%)$ & $0.008(1.7 \%)$ \\
\hline
\end{tabular}

approaches for NNs with non-traditional input data, which have been discussed in the literature [23].

\section{B. Synthesis Results}

We analyze both aforementioned networks for two configurations, with weights fixed at synthesis time and with variable weights (excl. storage, modeled as inputs). The fixed weights are taken from the aforementioned trained models.

We provide an overview of synthesis results for different configurations in Tbl. II. We synthesized both networks listed in Tbl. I in GlobalFoundries $22 \mathrm{~nm}$ SOI technology with LVT cells in the typical case corner at $0.65 \mathrm{~V}$ and $25^{\circ} \mathrm{C}$. The configuration with variable weights scales with the computational effort associated with the network $(1.13 \mathrm{MOp} / \mathrm{img}$ and $5.34 \mathrm{MOp} / \mathrm{img}$ for the $16 \times 16$ and $32 \times 32$ networks) with 0.97 and $0.92 \mathrm{MOp} / \mathrm{cycle} / \mathrm{mm}^{2}$, respectively. The variable parameters/weights configuration does not include the storage of the parameters themselves, which would add $1.60 \mu^{2}{ }^{2}(8.0 \mathrm{GE})$ per FF. This weight memory could be loaded through a scanchain without additional logic cells (from some flash memory elsewhere on the device). Alternatively, non-volatile memory cells could be used to store them. The number of parameters is 33 and $65 \mathrm{kbit}$ and thus $0.05 \mathrm{~mm}^{2}$ (264 kGE) and $0.10 \mathrm{~mm}^{2}$ $(520 \mathrm{kGE})$ for the $16 \times 16$ and $32 \times 32$ network, respectively.

Looking at the more detailed area breakdown in Tbl. III, we can see that there is a massive reduction when fixing the weights before synthesis. Clearly, this eliminates all the XNOR operations which become either inverters or wires, and even some of the inverters can now be shared among all units having this particular input value in their receptive field. However, based on the estimates described in Sec. III-A, this cannot explain all the savings. Additional cells can be saved through the reuse of identical partial results, which not only can occur randomly but must occur frequently. For example, consider 16 parallel popcount units summing over 8 values each. We can

TABLE IV

ENERGY AND LEAKAGE BREAKDOWN FOR THE $16 \times 16$ NETWORK

\begin{tabular}{|c|c|c|c|c|}
\hline \multirow[b]{2}{*}{ layer } & \multicolumn{2}{|c|}{ — var. weights } & \multicolumn{2}{|c|}{ _ fixed weights } \\
\hline & energy/img [pJ] & leakage & energy/img [pJ] & leakage \\
\hline 1 & $38(1.6 \%)$ & & $9(0.5 \%)$ & $8 \mu \mathrm{W}$ \\
\hline 2 & $806(33.7 \%)$ & $547 \mu \mathrm{W}$ & $478(28.5 \%)$ & $152 \mu \mathrm{W}$ \\
\hline 3 & $1440(60.2 \%)$ & $310 \mu \mathrm{W}$ & $1037(61.9 \%)$ & $163 \mu \mathrm{W}$ \\
\hline 4 & $107(4.5 \%)$ & $20 \mu \mathrm{W}$ & $151(9.0 \%)$ & $7 \mu \mathrm{W}$ \\
\hline
\end{tabular}


TABLE V

COMPARISON WITH STATE-OF-THE-ART APPROACHES FOR DEEP-LEARNING NEAR SENSOR PROCESSING

\begin{tabular}{|c|c|c|c|c|c|}
\hline Approach & {$[15]$} & [19] & XNOR-POP [21] & YodaNN [8] & This Work \\
\hline Description & $\begin{array}{l}\text { Analog switched- } \\
\text { capacitor matrix-mult }\end{array}$ & $\begin{array}{l}\text { Cluster of } 256 \mathrm{BNN} \\
\text { Digital Engines }\end{array}$ & $\begin{array}{l}\text { In-memory } \quad \text { XNOR } \\
\text { Engines }\end{array}$ & $\begin{array}{l}\text { Binary-weight digital } \\
\text { accelerator }\end{array}$ & $\begin{array}{l}\text { Combinational BNN } \\
\text { with fixed weights }\end{array}$ \\
\hline Technology & $40 \mathrm{~nm}$ & $14 \mathrm{~nm}$ & $32 \mathrm{~nm}$ (logic) & $65 \mathrm{~nm}$ & $22 \mathrm{~nm}$ SOI \\
\hline Peak Performance & $2 \mathrm{GOp} / \mathrm{s}$ & $16 \mathrm{TOp} / \mathrm{s}$ & $5.2 \mathrm{TOp} / \mathrm{s}$ & $1.5 \mathrm{TOp} / \mathrm{s}$ & $91.12 \mathrm{TOp} / \mathrm{s}$ \\
\hline Power Consumption & $228 \mu \mathrm{W} @ 1 \mathrm{~V}$ & - & $237 \mathrm{~mW}$ & $25 \mathrm{~mW} @ 0.6 \mathrm{~V}$ & 135 mW (dyn)@0.65 V \\
\hline Peak Energy-efficiency & $8.77 \mathrm{TOp} / \mathrm{s} / \mathrm{W}$ & - & $22.2 \mathrm{TOp} / \mathrm{s} / \mathrm{W}$ & $61.2 \mathrm{TOp} / \mathrm{s} / \mathrm{W}$ & $672.6 \mathrm{TOp} / \mathrm{s} / \mathrm{W}$ \\
\hline Area & - & - & $2.24 \mathrm{~mm}^{2}$ & $1.9 \mathrm{~mm}^{2}(1.33 \mathrm{MGE})$ & $0.46 \mathrm{~mm}^{2}(2.32 \mathrm{MGE})$ \\
\hline
\end{tabular}

split the value into 4 groups with 2 values each. Two binary values can generate $2^{2}=4$ output combinations. Since we have 16 units of which each will need one of the combinations, they will on average be reused 4 times. This is only possible with fixed weights, otherwise the values to reuse would have to be multiplexed, thereby loosing all the savings.

Generally, we can observe that these already small networks for low-resolution images require a sizable amount of area, such that more advanced ad-hoc synthesis tools exploiting the sharing of weights and intermediate results are needed.

\section{Energy Efficiency Evaluations}

We have performed post-synthesis power simulations using 100 randomly selected real images from the dataset as stimuli. The results are also reported in Tbl. II while a detailed perlayer breakdown is shown in Tbl. IV. We see that the $32 \times 32$ model has lower energy-efficiency and higher latency with fixed weights, opposed to the smaller model. We attribute this to the optimization towards minimal area without a timing constraint, which favors weak drivers and high-fanout nets with accordingly long transition times. We have observed that particularly many cells are driven by signals corresponding to the feature maps between layers, and that fixed-weight circuits are more affected by this due to the significantly higher input capacitance of FAs relative to XOR gates.

When heavily duty-cycling a device, leakage can become a problem. In this case, we see $945 \mu \mathrm{W}$ and $331 \mu \mathrm{W}$ of leakage power, which might be significant enough in case of low utilization to require mitigation through power-gating or using HVT cells. Generally, voltage scaling can also be applied, not only reducing leakage, but also active power dissipation. The throughput we observe in the range of $50 \mathrm{Mframe} / \mathrm{s}$ is far in excess of what is meaningful for most applications. Thus aggressive voltage scaling, power gating and the reverse body biasing available in this FD-SOI technology should be optimally combined to reach the minimum energy point where leakage and dynamic power are equal while the supply is ON.

A comparison with state-of-the-art approaches is reported in Tbl. V. The energy efficiency numbers of our approach are in the order of $10 \times$ higher than those of the next competitor YodaNN [8]. However, they are fundamentally different in the sense that YodaNN (a) runs the more complex binary weight networks, (b) requires additional off-chip memory for the weights and intermediate results, (c) can run large networks with a fixed-size accelerator, and (d) is in an older technology but doing aggressive voltage scaling.

We expect the energy values to be highly dependent on the input data, since energy is consumed only when values toggle. While a single pixel toggling at the input might affect many values later in the network, it has been shown that rather the opposite effect can be seen: changes at the input tend to vanish deeper into the network [24]. A purely

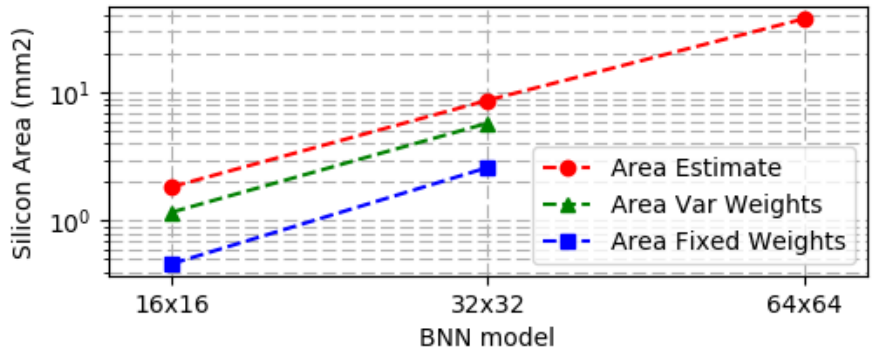

Fig. 3. Silicon area estimation (in red) and measurements with variable (green) and fixed (blue) weights of three BNNs featuring a model complexity which scales depending on the imager resolution. The area occupation of the $64 \times 64$ model is not reported because the synthesis tool is not able to handle such a complex and large design.

combinational implementation fully leverages this and BNNs naturally have a threshold that keeps small changes from propagating and might thus perform even better for many realworld applications.

\section{Scaling to Larger Networks}

Our results show an area requirement in the range of 2.05 to $2.46 \mathrm{GE} / \mathrm{Op}$ and an average $1.9 \mathrm{fJ} / \mathrm{Op}$. Scaling this up to $0.5 \mathrm{~cm}^{2}$ (250 MGE) of silicon and an energy consumption of only $210 \mathrm{~nJ} / \mathrm{img}$, we could map networks of around $110 \mathrm{MOp} / \mathrm{img}$ - this is already more than optimized highquality ImageNet classification networks such as ShuffleNets require [25].

Fig. 3 shows the estimation and measurements of the silicon area corresponding to the synthesized BNNs for fixed and variable weights. We also consider a model with a larger $64 \times 64$ input imager receptive field and a higher complexity (5 convolutional and 2 fully-connected layers, 23.05 GOp/img). Such a model presents is more accurate on the considered classification task $(73.6 \%)$ but current synthesis tool cannot handle the high complexity of the design, using in excess of $256 \mathrm{~GB}$ of memory. When estimating the area occupancy, the $64 \times 64$ BNNs result to be $4.3 \times$ larger than the area estimated for the $32 \times 32$ model. A direct optimization of such large designs is out of scope of today's EDA tools, clearly showing the need for specialized design automation tools for BNNs.

\section{CONCLUSiON}

We have presented a purely combinational design and synthesis of BNNs for near-sensor processing. Our results demonstrate the suitability and the energy efficiency benefits of the proposed solution, fitting on a silicon area of $2.61 \mathrm{~mm}^{2}$ when considering a BNN model with $32 \times 32$ binary input data and weight parameters fixed at design time. Our study also highlighted the need for novel synthesis tools able to deal with very large and complex network designs, that are not easily handled by current tools. 


\section{REFERENCES}

[1] M. Alioto, Enabling the Internet of Things: From Integrated Circuits to Integrated Systems. Springer, 2017.

[2] M. Rusci, D. Rossi et al., "An event-driven ultra-low-power smart visual sensor," IEEE Sensors Journal, vol. 16, no. 13, pp. 5344-5353, 2016.

[3] Á. Rodríguez-Vázquez, R. Carmona-Galán et al. "In the quest of visionsensors-on-chip: Pre-processing sensors for data reduction," Electronic Imaging, vol. 2017, no. 11, pp. 96-101, 2017.

[4] R. LiKamWa, Y. Hou et al., "Redeye: analog convnet image sensor architecture for continuous mobile vision," in Proc. IEEE ISCA, 2016, pp. $255-266$.

[5] S. Zhang, M. Kang et al., "Reducing the energy cost of inference via in-sensor information processing," arXiv:1607.00667, 2016.

[6] J. Fernández-Berni, R. Carmona-Galán et al., "Focal-plane sensingprocessing: A power-efficient approach for the implementation of privacy-aware networked visual sensors," Sensors, vol. 14, no. 8, pp. $15203-15226,2014$

[7] M. Gottardi, N. Massari, and S. A. Jawed, "A $100 \mu$ w $128 \times 64$ pixels contrast-based asynchronous binary vision sensor for sensor networks applications," IEEE Journal of Solid-State Circuits, vol. 44, no. 5, pp. $1582-1592,2009$.

[8] R. Andri, L. Cavigelli et al., "Yodann: An architecture for ultralow power binary-weight cnn acceleration," IEEE Transactions on Computer-Aided Design of Integrated Circuits and Systems, 2017.

[9] M. Courbariaux, I. Hubara et al., "Binarized neural networks: Training deep neural networks with weights and activations constrained to+ 1 or-1," arXiv:1602.02830, 2016.

[10] M. Rastegari, V. Ordonez et al., "Xnor-net: Imagenet classification using binary convolutional neural networks," in Proc. ECCV. Springer, 2016, pp. $525-542$.

[11] Y. Umuroglu, N. J. Fraser et al., "Finn: A framework for fast, scalable binarized neural network inference," in Proc. ACM/SIGDA FPGA, 2017, pp. $65-74$

[12] J. Choi, S. Park et al., "A 3.4- $\mu$ w object-adaptive cmos image sensor with embedded feature extraction algorithm for motion-triggered objectof-interest imaging," IEEE Journal of Solid-State Circuits, vol. 49, no. 1, pp. 289-300, 2014

[13] G. Kim, M. Barangi et al., "A 467nw cmos visual motion sensor with temporal averaging and pixel aggregation," in Proc. IEEE ISSCC, 2013, pp. $480-481$.

[14] H. G. Chen, S. Jayasuriya et al. "Asp vision: Optically computing the first layer of convolutional neural networks using angle sensitive pixels," in Proc. IEEE CVPR, 2016, pp. 903-912.

[15] E. H. Lee and S. S. Wong, "Analysis and design of a passive switchedcapacitor matrix multiplier for approximate computing," IEEE Journal of Solid-State Circuits, vol. 52, no. 1, pp. 261-271, 2017.

[16] Z. Du, R. Fasthuber et al., "Shidiannao: Shifting vision processing closer to the sensor," in ACM SIGARCH Computer Architecture News, vol. 43 , no. 3, 2015, pp. 92-104.

[17] L. Cavigelli and L. Benini, "Origami: A 803-gop/s/w convolutional network accelerator," IEEE Transactions on Circuits and Systems for Video Technology, vol. 27, no. 11, pp. 2461-2475, 2017.

[18] V. Sze, Y.-H. Chen et al., "Efficient processing of deep neural networks: A tutorial and survey," arXiv:1703.09039, 2017.

[19] E. Nurvitadhi, D. Sheffield et al., "Accelerating binarized neural networks: Comparison of fpga, cpu, gpu, and asic," in Proc. FPT, 2016, pp. $77-84$.

[20] K. Ando, K. Ueyoshi et al., "Brein memory: A 13-layer 4.2 k neuron/0.8 $\mathrm{m}$ synapse binary/ternary reconfigurable in-memory deep neural network accelerator in $65 \mathrm{~nm}$ cmos," in Proc. VLSI Symposium, 2017.

[21] L. Jiang, M. Kim et al., "Xnor-pop: A processing-in-memory architecture for binary convolutional neural networks in wide-io2 drams," in Proc. IEEE/ACM ISLPED, 2017.

[22] "The traffic surveillance workshop and challenge 2017 (tswc- 2017)," 2017, MIO-TCD: MIOvision Traffic Camera Dataset. [Online]. Available: http://podoce.dinf.usherbrooke.ca

[23] S. Jayasuriya, O. Gallo et al., "Deep learning with energy-efficient binary gradient cameras," arXiv:1612.00986, 2016.

[24] L. Cavigelli, P. Degen, and L. Benini, "Cbinfer: Change-based inference for convolutional neural networks on video data," arXiv:1704.04313, 2017.

[25] X. Zhang, X. Zhou et al., "Shufflenet: An extremely efficient convolutional neural network for mobile devices," arXiv:1707.01083, 2017. 